\title{
A Novel and Simple Technique of Reconstructing the Central Arch Mandibular Defects-a Solution During the Resource-Constrained Setting of COVID Crisis
}

\author{
Shiv Rajan ${ }^{1} \cdot$ Naseem Akhtar ${ }^{1}$ (D) $\cdot$ Vijay Kumar $^{1} \cdot$ Sameer Gupta $^{1} \cdot$ Sanjeev Misra ${ }^{2} \cdot$ Arun Chaturvedi $^{1}$ • \\ Puneet Prakash ${ }^{1} \cdot$ Tashbihul Azhar $^{1}$
}

Received: 22 May 2020 / Accepted: 15 September 2020 /Published online: 25 September 2020

(C) Indian Association of Surgical Oncology 2020

\begin{abstract}
The current COVID 19 pandemic has a major impact on healthcare delivery globally. Oral cancer involving anterior arch of mandible is difficult to reconstruct and ideally, requires free fibular osteomyocutaneous flap. During this time of resource constraint situation, these free flaps are not a great choice, as it increases exposure of both patient and surgical team to the deadly virus. We are describing a novel method of reconstruction after resection of oral cancer involving anterior arch of mandible. In this new technique, we have reconstructed central arch defect by hanging bipaddle pectoralis major myocutaneous flap with orbicularis oris muscle using ethylene terephthalate suture. Operative time, early postoperative complications and early cosmetic and functional outcome were assessed. We have used this novel technique in eight patients of T4a oral cancer involving anterior arch of mandible and skin over chin. Mean operative time was $180 \mathrm{~min}$. One patient had minor flap loss with surgical site infection (Clavien-Dindo grade I). In all patients, we were able to discharge all patients on eighth postoperative day. Cosmetic outcome and functional outcomes were mostly satisfactory. All patients were able to oppose their lips without any oral incompetence and drooling. Tongue mobility was good. There was no incidence of 'Andy Gump deformity'. This is a feasible option for reconstructing anterior arch defect in resource- and time-limited setting of COVID 19 pandemic. This technique can also be used in comorbid conditions where it is not advisable to do very long surgery.
\end{abstract}

Keywords Ethylene terephthalate $\cdot$ COVID $19 \cdot$ Andy Gump deformity · Pectoralis major myocutaneous flap · Free fibular osteomyocutaneous flap $\cdot$ Anterior arch mandible

\section{Introduction}

Oral cancer is one of the commonest cancer in India [1]. A large number of patients present in an advanced stage with involvement of mandible and overlying skin. These kinds of tumours involving the anterior arch of the mandible are difficult to reconstruct and usually require vascularized free fibular osteocutaneous flap $[2,3]$.
Naseem Akhtar

naseemakhtar97@gmail.com

Shiv Rajan

shivrajan.194@gmail.com

Vijay Kumar

drvkumar2007@gmail.com

Sameer Gupta

sameerdr79@gmail.com

Sanjeev Misra

misralko@gmail.com
Arun Chaturvedi

drchatur@gmail.com

Puneet Prakash

drpuneetsinha@gmail.com

Tashbihul Azhar

tashbihmariam@gmail.com

1 Department of Surgical Oncology, King George's Medical University, Lucknow, UP, India

2 All India Institute of Medical Sciences, Jodhpur, Rajasthan, India 
COVID 19 (SARS-CoV-2) pandemic poses unique challenges for the cancer patients as well as health care providers. Cancer patients are immunocompromised and have a high chance of getting infected while attending the hospital $[4,5]$. But, on the other hand, cancer needs to be treated, on a priority basis. In this situation, we need a surgical solution which is less cumbersome, requires less operative time and hospitalization with satisfactory functional outcome.
Vascularized free fibular osteocutaneous flap is difficult and time-consuming surgery. The patient is required to stay for long duration in hospital after surgery. It would not be a great choice during a resource-constrained setting or when experts are not available at the centre and patient is not able to travel due to pandemic.

We are describing a unique way of reconstructing central arch by hanging bipaddle pectoralis major myocutaneous flap
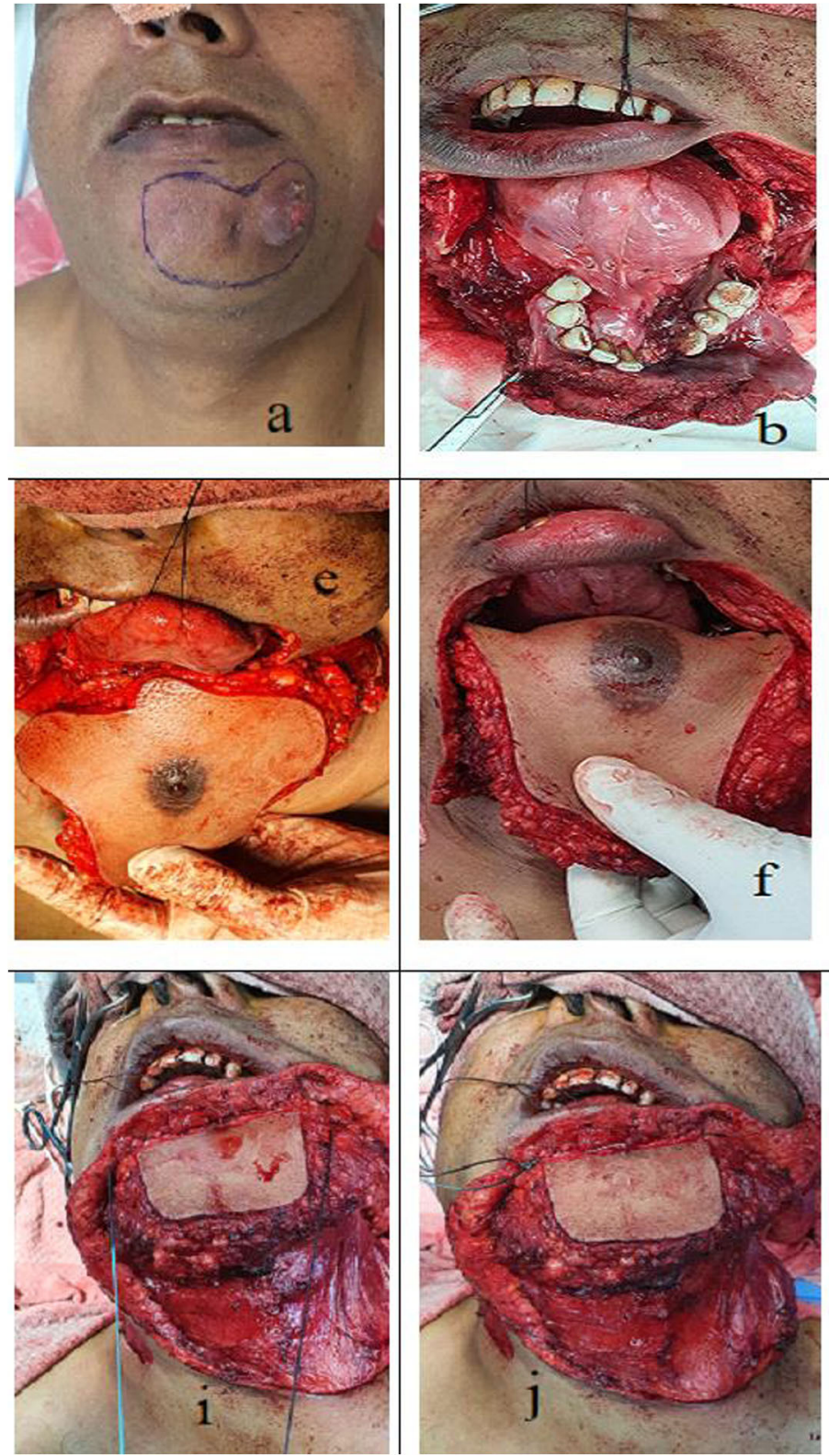

Fig. 1 Operative technique. a Primary tumour with chin involvement. b Mucosal cuts. $\mathbf{c}$ Defect after resection. d Marking of bipaddle pectoralis major flap. e Flap brought to the defect. f Inner paddle of the flap sutured to mucosa. g Flap underlying the lower lip de-epithelized. h Inner epidermal edge of the flap (inner paddle) sutured with inner mucosal
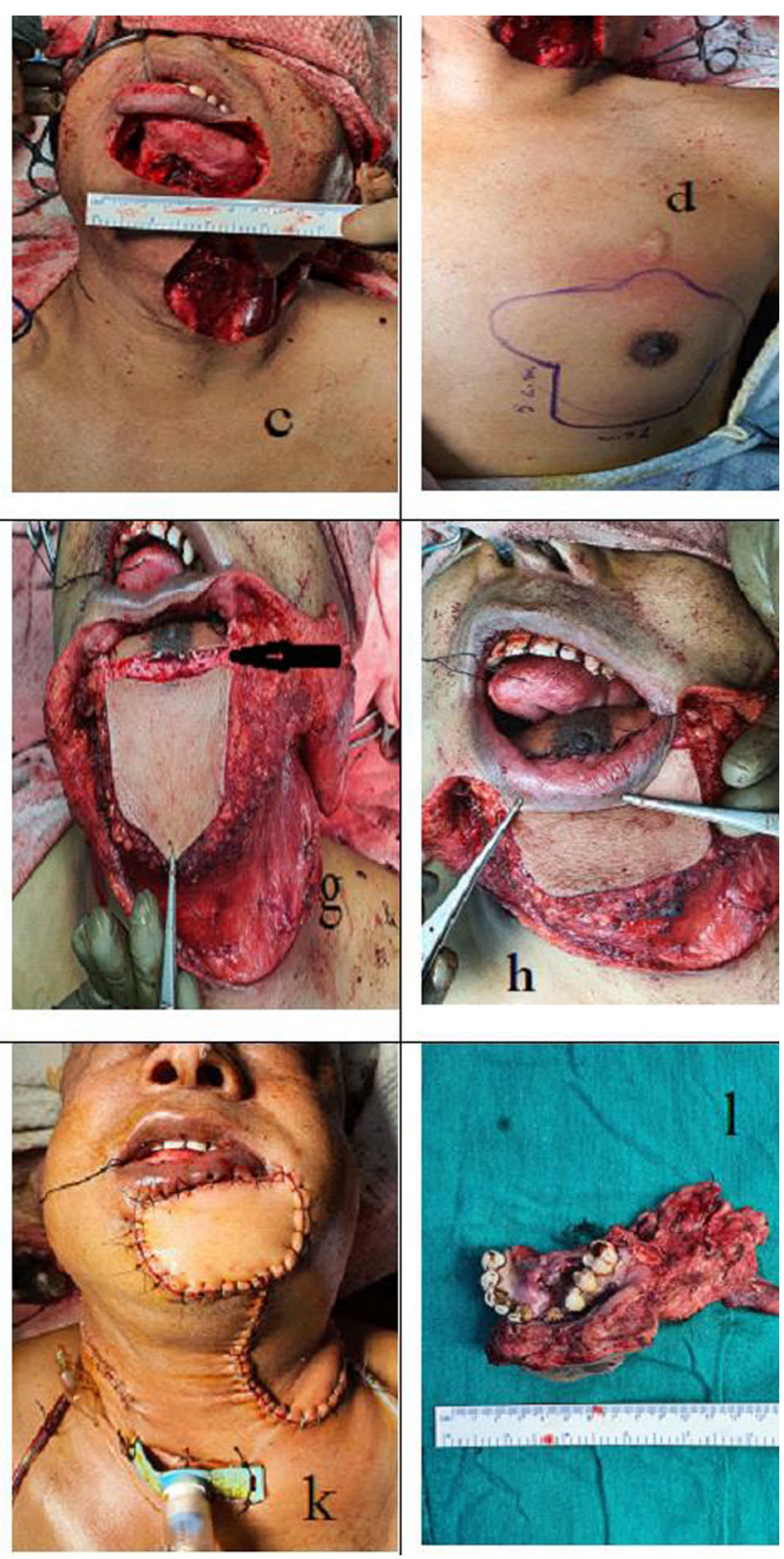

edge of the lip. i Ethylene terephthalate suture passed through the orbicularis oris muscle all along lower lip. $\mathbf{j}$ Same suture passed through the exposed dermis of the flap to tie the knot at the end. $\mathbf{k}$ Edge of epidermis towards outer paddle was sutured to skin of lower lip. I Resected specimen 
(PMMC) with orbicularis oris muscle using ethylene terephthalate suture.

\section{Method}

We are reporting eight patients in which we have used a novel technique of reconstruction. We selected patients of squamous cell carcinoma of the anterior arch in whom a rim of lower lip along with orbicularis oris muscle was left intact following resection. In our technique of reconstruction, intact orbicularis oris muscle is a prerequisite for reconstruction (Fig. 1a).

\section{Technique}

In this technique, we use poly-ethylene terephthalate nonabsorbable braided suture no. 5 [6]. It is prepared from fibres of high molecular weight, long chain and linear polyesters having recurrent aromatic rings as an integral component. It is dyed green to enhance visibility.

Following standard resection of primary tumour which included central segmental mandibulectomy with bilateral neck dissection, the surgical defect was measured. Bipaddle PMMC flap based on thoraco-acromial artery was marked. The flap was then harvested and tunnelled through the neck to bring it at the site of the defect. Inner paddle of the flap was sutured to the remaining mucosa of the floor of mouth and ventral surface of tongue. A $5 \mathrm{~mm}$ width of flap underlying the lower lip was de-epithelized. This marked the junction of inner and outer paddle of the flap. The inner epidermal edge of the flap was (inner paddle) sutured with inner mucosal edge of the lip. Ethylene terephthalate suture was then loaded over a needle and passed through the orbicularis oris muscle all along lower lip, and then, this suture was turned back and passed through the exposed dermis of the flap to bring it out near the point of first entry of suture and knot was tied there. In this way, flap was suspended on orbicularis oris muscle at the junction of its inner and outer paddle. Edge of epidermis towards outer paddle was sutured to skin of lower lip. In the final step, the outer edge of external paddle was sutured to cover the skin defect (Fig. 1). Temporary tracheostomy was done in all cases which was removed after $72 \mathrm{~h}$. A nasogastric tube was placed on the first postoperative day for feeding. It was removed after at least 6 days after ensuring proper oral intake and absence of any oro-cutaneous fistula.

Total operative time and early postoperative complications were recorded. All patients were discharged on 8th postoperative day. At the time of discharge, all cases were assessed for cosmetic and functional outcome based on subjective scale (good, satisfactory, poor) (Fig. 2).
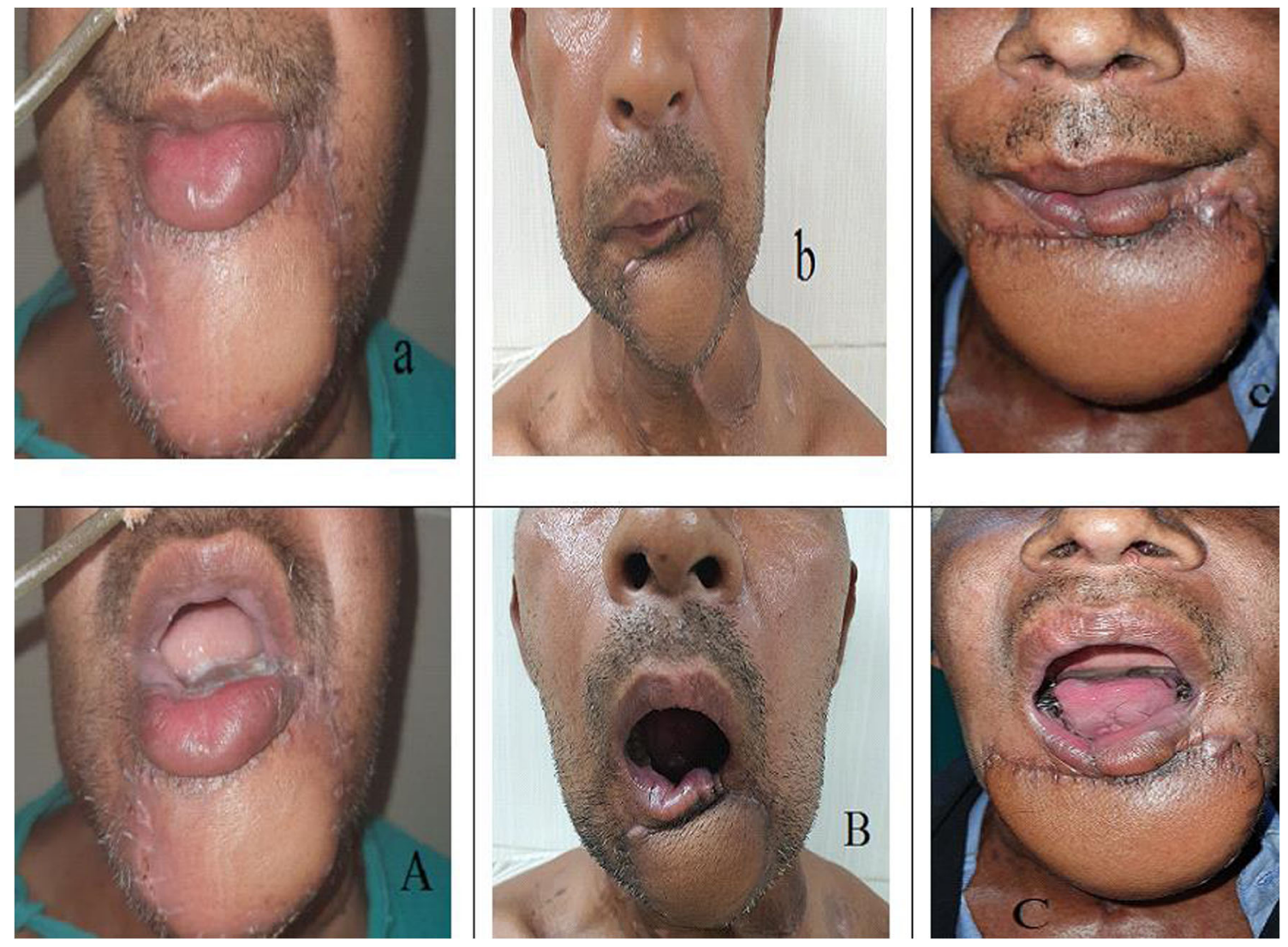

Fig. 2 Final outcome. a-c Closed mouth appearance. A-C Open mouth appearance of same patients 


\section{Results}

We have used this novel technique in eight patients. Median age of patients was 50 years (30-68). Six were male and two females. All patients had stage IVA disease. Seven patients had disease primarily in lower alveolus, and one was having disease of mucosal lip extending to gingivolabial sulcus. Mean operative time was $180 \mathrm{~min}$. In all cases, wide excision of primary was done along with central segmental mandibulectomy and excision of involved skin along with adequate margin. In all cases, the neck was addressed on both sides. Postoperatively, there was no bleeding, wound dehiscence, orocutaneous fistula or major flap loss. One patient had minor flap loss with surgical site infection (Clavien-Dindo grade I).

All patients received adjuvant radiotherapy. Cosmetic and functional outcomes were assessed after completion of radiotherapy. Cosmetic outcome was good in five patients on subjective scale. Functional outcome was rated as good by six and satisfactory by two patients (Table 1). All eight patients were able to oppose their lips without any oral incompetence (Fig. 2). There was good tongue mobility in all cases. There was no incidence of 'Andy Gump deformity'.

\section{Discussion}

Defect after resection of cancer involving anterior arch of mandible poses unique challenges in reconstruction. Ideal reconstruction in usual situations is vascularized free fibular osteocutaneous flap [2,3]. But hospitals are busy with treatment of COVID patients and surgical units are functioning in limited way. It is difficult to perform prolonged surgeries especially with anaesthetist busy with intensive care of COVID patient. Latest data on microvascular flap reconstruction in head and neck oncology shows variable practice pattern in academic institution across USA during this pandemic [7]. Reconstruction using metallic plate is not suitable for anterior arch as it has a high extrusion rate.

Till now, we have no idea about how long this pandemic may continue. What we are expecting from trends all over world is that this issue is not going to be solved in at least 3

Table 1 Cosmetic and functional outcome of reconstruction

\begin{tabular}{ll}
\hline Cosmetic outcome & Number (\%) \\
Good & $5(62.5 \%)$ \\
Satisfactory & $3(37.5 \%)$ \\
Poor & $0(0 \%)$ \\
Functional outcome & Number (\%) \\
Good & $6(75 \%)$ \\
Satisfactory & $2(25 \%)$ \\
Poor & $0(0 \%)$ \\
\hline
\end{tabular}

or 4 months. Treatment of any cancer is a semi-emergency procedure which cannot be done after 4 months. Using only soft tissue for reconstruction of anterior arch leads to sagging of the flap with an ugly 'Andy Gump deformity'.

This method of reconstruction is done by oncosurgeons themselves requiring fewer people to be exposed and less crowding in operation room. This is fast and the operative time is less than the free fibular osteocutaneous flap (180 $\mathrm{min}$ vs $474 \mathrm{~min})[8,9]$.

As this reconstruction is based on pedicled flap, chances of flap failure is less compared with free fibular osteocutaneous flap. In this technique, patients were mobilized early as compared with free fibular osteocutaneous flap (day 1 vs days 5 to 24); recovery is fast and can be sent home quickly, thus reducing exposure of the patient, as well as health care workers [2]. Cosmetic outcomes of this flap were comparable with free fibular osteocutaneous flap. Functional outcomes are satisfactory.

There are certain limitations to this study. At present, we do not know the long-term cosmetic and functional outcomes of these techniques.

\section{Conclusion}

This innovative technique is a feasible option for reconstructing anterior arch defect without compromising on oncological safety especially during COVID 19 pandemic. This technique can also be used in comorbid conditions where it is not advisable to do very long surgery. Future long-term outcomes are to be seen and will be analysed subsequently.

Acknowledgements We would like to thank our Vice Chancellor for his continuous academic support.

\section{Compliance with Ethical Standards}

Conflict of Interest The authors declare that they have no conflict of interest.

Ethical Approval All procedures performed in studies involving human participants were in accordance with the ethical standards of the institutional and/or national research committee and with the 1964 Helsinki declaration and its later amendments or comparable ethical standards.

Informed Consent Informed consent was obtained from all individual participants included in the study.

\section{References}

1. Bray F, Ferlay J, Soerjomataram I, Siegel RL, Torre LA, Jemal A (2018) Global cancer statistics 2018: GLOBOCAN estimates of incidence and mortality worldwide for 36 cancers in 185 countries. CA Cancer J Clin 68:394-424 
2. Hidalgo DA (1989) Fibular free flap: a new method of mandible reconstruction. Plast Reconstr Surg 84(1):71-79

3. Inbal A, Gur E, Zaretski A, Barnea Y, Khafif A, Amir A (2015) The "origami" composite free fibula flap for complex defects of the mandible, floor of the mouth, and tongue. J Oral Maxillofac Surg 73(8): $1617-1626$

4. Chambers WH, Rabinowich H, Herberman RB (2003) Mechanisms of immunosuppression. In: Kufe DW, Pollock RE, Weichselbaum RR et al (eds) Holland-Frei Cancer Medicine, 6th edn. BC Decker, Hamilton

5. Liang W, Guan W, Chen R, Wang W, Li J, Xu K, Li C, Ai Q, Lu W, Liang H, Li S, He J (2020) Cancer patients in SARS-CoV-2 infection: a nationwide analysis in China. Lancet Oncol 21(3):335-337. https://doi.org/10.1016/S1470-2045(20)30096-6

6. Byrne M, Aly A (2019) The surgical suture. Aesthet Surg J 39(Supplement_2):S67-S72
7. Patel RJ, Kejner A, McMullen C (2020) Early institutional head and neck oncologic and microvascular surgery practice patterns across the United States during the SARS-CoV-2 (COVID19) pandemic. Head Neck 42(6):1168-1172

8. Yamamoto N, Morikawa T, Yakushiji T, Shibahara T (2018) Mandibular reconstruction with free vascularized fibular graft. Bull Tokyo Dent Coll 59(4):299-311

9. Mazzola F, Smithers F, Cheng K et al (2020) Time and cost-analysis of virtual surgical planning for head and neck reconstruction: a matched pair analysis. Oral Oncol 100:104491

Publisher's Note Springer Nature remains neutral with regard to jurisdictional claims in published maps and institutional affiliations. 